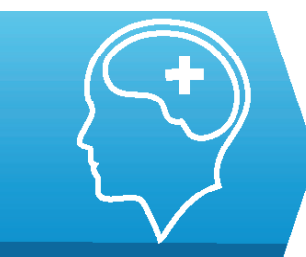

PSYCHIATRY
1) General Department, University of Thessaly, Larissa, Greece

2) Emergency Department, General Hospital of Karditsa, Karditsa, Greece

3) Health Center of Sofades-Karditsa, Karditsa, Greece

4) Department of Nursing, University of Peloponnese, Sparta, Greece

5) Nursing Department, International University of Greece, Thessaloniki, Greece

6) Psychiatric Department, "Sotiria" Athens General Hospital for Chest Diseases, Athens, Greece

\title{
Depression, anxiety and stress among patients with hematological malignancies and the association with quality of life: a cross-sectional study
}

Ioanna V. Papathanasiou ${ }^{1}$, Konstantinos Kelepouris ${ }^{2}$, Chrisoula Valari ${ }^{3}$, Dimitrios Papagiannis $^{1}$, Foteini Tzavella ${ }^{4}$, Lambrini Kourkouta ${ }^{5}$, Konstantinos Tsaras ${ }^{1}$, Evangelos C. Fradelos ${ }^{4,6}$

\begin{abstract}
Aim. To evaluate psychological distress and quality of life of patients with hematological malignancies, as well as to identify the prognostic factors that aggravate their condition.

Methods. A cross-sectional, descriptive study including 87 patients with Hematological Malignancies was conducted. Data were collected with an anonymous questionnaire consisted by A) a sheet with socio-demographic characteristics, B) the Depression, Stress and Stress Scale (DASS21), and C) World Health Organization Life Quality Scale (WHOQOL - BREF30). The processing and statistical analysis of the empirical material of the research were done using the SPSS (Statistical Package for Social Science) 22.0.

Results. The results showed that the age of the sample averaged 71.22 years $(\mathrm{SD}=8.71)$. Regarding their marital status, single mothers were $9.2 \%$, married $62.1 \%$. With regard to disease-related features, $28.7 \%$ of patients had Hodgkin's Lymphoma, 27.6\% Chronic Lymphocytic Leukemia, 27.6\% MDL and 16.1\% of patients had myelodysplastic syndrome Multiple myeloma. In the investigation of the relationship between the Mental health Scale and the Quality of Life, negative correlations of Depression, Stress, Stress and the total DAS Scale with all dimensions of Patient Quality of Life were found.
\end{abstract}

Conclusions. Higher levels of psychological distress and poor mental health are associated with a low level of quality of life.

Keywords: hematologic malignancies, psychological distress, quality of life, patients

\section{Introduction}

Hematologic malignancies are common malignant disorders. Their prevalence has increased significantly in recent decades, especially in developed countries and, therefore, they are a significant health problem with marked consequences for society and the economy. In the Western population, a wealth of evidence demonstrates that hematologic malignancies have adverse effects in a variety of domains, including quality of life, physical and mental health, and healthcare-related costs $[1,2]$.
Pain intensity and reduced functionality, in conjunction with differing severity/chronicity of problems, can have a significant impact on the patient's overall well-being, as they can aggravate physical and psychological health and degrade the quality of life. "Quality of Life" is one of the most widely used indicators for assessing therapeutic interventions and strategies that aim to improve mental health. "Quality of Life" has also been extensively researched in studies on patients with severe health conditions and studies on chronic pain [3]. 
Patients' quality of life is evaluated using psychometric tests, mainly questionnaires. Most measuring instruments provide a holistic, overarching approach to quality-of-life, which includes assessing a number of different aspects, such as social, family and work functioning, psychological well-being as well as environmental factors that may affect, at least to some degree, an individual's quality of life, such as physical environment, financial resources, educational attainment and employment opportunities [4,5].

Therefore, the study of psychosocial factors influencing the course and the experience of the severe disease has received increased attention, which is associated with the increasing acceptance of the biopsychosocial (BPS) model of health, in the scientific and in the clinical community. Psycho-oncology, the field examining cancer through the lens of psychology, suggests that most cancer patients deal with numerous personal and interpersonal problems and fears that have been called the 6 D's [6]. These issues include discomfort, dependency, disability, disfigurement, disruption and death. Studies examining QoL in hematological cancer patients revealed that those patients experience several burdens. Specifically, a review of Allart-Vorelli et al. (2015), based on various quality-of-life assessment tools results, pointed out that hematological cancer patients appear to have lower QoL in the physical, psychological, emotional and social domains compared with the general population. Unsurprisingly, patients with hematologic malignancies exhibit lower overall health and extremely high levels of fatigue and pain, which are common features of such medical conditions. Patients also show cognitive impairments, such as problems with memory and attention, increased levels of anxiety and depression and decreased sexual activity, which is also linked to the development of a negative body image [7]. Their relationships with family members, although in many cases are likely to be strengthened, are characterized by high-stress levels, uncertainty and fear, while the person's friendly relationships may also be disrupted. These individuals also face work-related and financial challenges which are associated with the suppression caused by their disease and with the fact that hematologic cancer treatment is often expensive and takes place, almost exclusively, in urban areas, forcing patients and probably family members to temporarily change their residence from one place to another place $[8,9]$.

Most psychosocial research data on hematological malignancies come from quantitative research.

The purpose of this study was to investigate mental impairment and quality of life among patients with hematologic malignancies and the possible relationships between them. This study is a Descriptive Correlational Study using a cross-sectional design.

\section{Material and methods}

The study population includes patients with hematologic malignancies. In total, our sample consisted of $n=87$ with hematologic malignancies regardless of 'diagnosis', visiting the Hematology Department of the General Hospital of Karditsa for periodic, scheduled or non-scheduled examination and treatment. No exclusion criteria were implemented except cooperation and acceptance to participate in the study. It should be noted that patients with hematological malignancies managed in the General Hospital of Karditsa, were middle-aged and elderly patients, aged 45 years and over, as younger patients are referred to more specialized hospitals.

Non-Probability sampling was applied in this study, namely, convenience sampling.

The research material was collected using a fully structured questionnaire which consisted of three parts as follows:

A. Individual Characteristics Form. It included questions related to patients' sociodemographic features (gender, age, marital status, number of children, educational level, occupation, area of permanent residence, living alone or with others) and the characteristics associated with the disease (type of disease, years from diagnosis of disease, parenteral drug administration, cause of hospital admission, frequency of hospital treatment).

B. Depression, Anxiety and Stress Scale (DASS21). The Depression Anxiety and Stress Scale-21 (DASS) developed by Lovibond and Lovibond (1995) were used to assess patients' psychological distress [10]. This scale assesses mental health parameters that characterize a negative emotional state, namely depression, anxiety, and stress. DASS scale was used in this study as adapted in Greek by Lyrakos et al 2011. According to the authors, the Depression Anxiety Stress Scale has good construct and content validity while the internal consistency reliability and the split-half reliability are satisfactory [11].

The Depression Anxiety Stress Scale is a selfreferencing questionnaire, which has been used in a variety of research approaches to measure the negative emotional states of depression, anxiety and stress. The scale consists of 21 statements and is divided into three sub-scales that each has seven items: depression, anxiety, and stress. The responses are given on a 4-point Likert scale, ranging from zero ("did not apply to me at all"), 1 ("applied to me to some degree, or some of the time"), 2 ("applied to me to a considerable degree, or a good part of time") to 3 ("applied to me very much or most of the time"). The total scale and subscale score are calculated by summing responses to the individual items multiplied by 2 . Therefore, sum scores for each of the subscales may range between 0 and 42 , while sum scores for the total DASS-total scale range between 0 and 126. Higher scores indicate high levels of negative emotional state and high levels of mental stress.

C. The World Health Organization (WHO) quality of life instrument (WHOQOL - BREF30). To estimate 
patient's health-related quality of life, we used the World Health Organization Quality of Life, WHOQOL - BREF questionnaire. The full-length WHOQOL was originally developed in 1994 by the World Health Organization aiming to develop an integrated QOL measurement tool suitable for cross-cultural comparison in diseased and healthy individuals [12]. The WHOQOL-BREF Greek version, which was used in the present study, was developed by Ginieri-Coccossis M, Triantafillou E, Tomaras V, Soldatos C, Mavreas V and Christodoulou G [13]. The results of the psychometric properties of the WHOQOLBREF Greek version showed that the questionnaire had a satisfactory level of internal consistency reliability, testretest reliability, construct validity, convergent validity and discriminant validity. According to the authors, the questionnaire is a valid and reliable instrument for measuring quality of life which can be used in the broader health sector to measure the quality of life of clinical groups and healthy individuals. The WHOQOL-BREF Greek version contains a total of 30 questions, divided into four broad domains - subscales, namely, physical health (nine items), psychological health (six items), social relations (five items) and environment (eight items) and four additional items derived from the cultural adaptation. The first two questions evaluate quality of life in general and satisfaction with health and constitute the domain "global quality of life/general health". Responses are rated on a 5-point Likert scale ranging from 1 ("very poor or not at all or very dissatisfied or never") to 5 ("very good or extremely or very satisfied or always"). Three questions are reverse scored. Total score of each domain is calculated from the average of the sum of the questions constituting each subscale, multiplied by 4 . Thus, potential scores for each domain range from 4 to 20. Higher scores indicate a higher quality of life in each particular domain.

The collection of research data was carried out at the Department of Hematology of the Karditsa General Hospital, from January to March 2018. The questionnaire was provided to patients during their stay at the Department, and after proper instructions, participants either completed the questionnaire by themselves or those who were unable to complete the questionnaire by themselves were interviewed. Participation in this research was voluntary, anonymous and confidential, and all research ethics followed.

Statistical analysis was performed with SPSS Version 22.0 statistic software package, using both descriptive and inferential statistics. In particular, the descriptive analysis included the frequency distribution for the qualitative variables (absolute- and relative frequencies) as well measures of dispersion and position for quantitative variables (mean, standard deviation, median, maximum and minimum values). Inferential statistics such as independent-samples t-test, one-way
ANOVA for independent samples (LSD method was used for multiple comparisons) as well as of Pearson correlation coefficient (r). DASS and WHOQOL-BREF scores were used as outcome measures of the studied relationships. For statistical analysis, a significant level of $\mathrm{p}<0.05$ was set.

\section{Results}

Regarding sociodemographic characteristics of the sample (Table I), 59.8\% were male, and $40.2 \%$ were female. Participants ranged from 49 years old to 89 years old, with an average age of 71.22 years old $(\mathrm{SD}=8.71)$. With regard to their marital status, 9.2\% were single, $62.1 \%$ were married, $8.0 \%$ were divorced, and $20.7 \%$ were widowed, while the majority of the sample (51.7\%) had 1 to 2 children. $46.0 \%$ had completed primary education, $33.3 \%$ had completed secondary education, and $20.7 \%$ had completed tertiary education. As regards patient's current or past jobs and occupations $33.3 \%$ were civil servants, $12.6 \%$ were private-sector employees, $32.2 \%$ were freelancers/ farmers and $21.8 \%$ were engaged in domestic duties. Most of the patients (63.2\%) lived in semirural or rural areas, while nearly three-quarters of participants reported living with family members.

As regards medical characteristics of the patients (Table I), 28.7\% suffered from Hodgkin's lymphoma, $27.6 \%$ from chronic lymphogenous leukaemia, $27.6 \%$ from Myelodysplastic syndrome (MDS) and 16.1\% from multiple myeloma. Years from diagnosis ranged from 0.5 to 10 years, with an average of 3.44 years $(\mathrm{SD}=2.34)$. $44.8 \%$ of participants received parenteral medications. The majority of the patients $(89.7 \%)$ went to the hospital for a scheduled examination, and the frequency of hospital treatment was monthly $(78.2 \%)$.

Internal consistency reliability of the DASS scale, using Cronbach's alpha, were as follows: overall scale (Alpha $=0.91$ ), depression (Alpha $=0.93$ ), anxiety (Alpha $=0.91)$ and stress (Alpha $=0.91)$. The fact that Cronbach alpha values were greater than 0.70 , indicates that all items used had high internal consistency (Table II).

The scores for the total DASS scale ranged between 0 and 126 , with a mean score of $45.31(\mathrm{SD}=31.29)$. Half of the patients scored below 40 (median $=40.00$ ), a score less than 63 which is the midpoint of the response scale, indicating that the majority of patients showed relatively low levels of total psychological stress (Table II).

Regarding scores for each DASS subscale, depression subscale score ranged from 0 to 42 , with a mean score of $15.15(\mathrm{SD}=11.82)$, anxiety subscale score ranged from 0 to 42 , with a mean score of 12.62 $(\mathrm{SD}=10.33)$ and stress subscale score ranged from 0 to 42 , with a mean score of $17.54(\mathrm{SD}=10.54)$. Stress subscale had the highest mean score followed by depression and anxiety subscales (Table II). 
Table I. Socio-demographic and medical characteristics of the patients $(n=87)$.

\begin{tabular}{|c|c|c|c|}
\hline Characteristics & & $\mathbf{n}$ & $\%$ \\
\hline \multirow{2}{*}{ Gender } & Male & 52 & 59.8 \\
\hline & Female & 35 & 40.2 \\
\hline \multirow{5}{*}{ Age (years) } & $50-59$ & 9 & 10.3 \\
\hline & $60-69$ & 26 & 29.9 \\
\hline & $70-79$ & 35 & 40.2 \\
\hline & $\geq 80$ & 17 & 19.5 \\
\hline & Mean \pm SD & \multicolumn{2}{|c|}{$71.22 \pm 8.71$} \\
\hline \multirow{2}{*}{ Current marital status } & Married & 54 & 62.1 \\
\hline & Not married & 33 & 37.9 \\
\hline \multirow{3}{*}{ Number of children } & 0 & 11 & 12.6 \\
\hline & $1-2$ & 45 & 51.7 \\
\hline & $\geq 3$ & 31 & 35.6 \\
\hline \multirow{3}{*}{ Highest level of education } & Primary & 40 & 46.0 \\
\hline & Secondary & 29 & 33.3 \\
\hline & Tertiary & 18 & 20.7 \\
\hline \multirow{4}{*}{ Employment status } & Public sector employee & 29 & 33.3 \\
\hline & Private sector employee & 11 & 12.6 \\
\hline & Freelancer & 28 & 32.2 \\
\hline & Household & 19 & 21.8 \\
\hline \multirow{3}{*}{ Place of residence } & Urban & 32 & 36.8 \\
\hline & Semi-urban & 22 & 25.3 \\
\hline & Rural & 33 & 37.9 \\
\hline \multirow{2}{*}{ Living arrangement } & Alone & 21 & 24.1 \\
\hline & With family & 66 & 75.9 \\
\hline \multirow{4}{*}{ Disease } & $\begin{array}{l}\text { Chronic Lymphogenous } \\
\text { Leukemia }\end{array}$ & 24 & 27.6 \\
\hline & Hodgkin's lymphoma & 25 & 28.7 \\
\hline & Multiple myeloma & 14 & 16.1 \\
\hline & Myelodysplastic syndrome & 24 & 27.6 \\
\hline \multirow{3}{*}{ Duration of disease (years) } & $\leq 5$ & 69 & 79.3 \\
\hline & $>5$ & 18 & 20.7 \\
\hline & Mean \pm SD & \multicolumn{2}{|c|}{$3.44 \pm 2.34$} \\
\hline
\end{tabular}

Table II. Scores of the WHOQOL-BREF (30-items Greek version) and DASS (21-items) subscales among the patients (n=87).

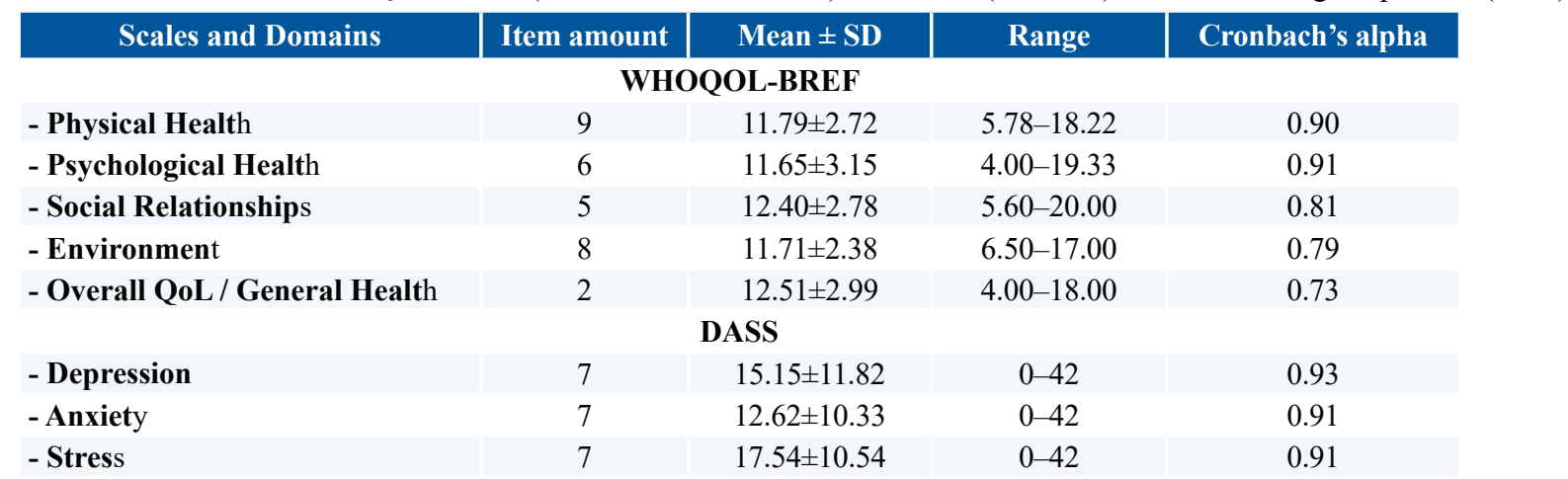

WHOQOL: World Health Organization Quality of Life, DASS: Depression Anxiety Stress Scale 
Table III. Correlation between WHOQOL-BREF dimensions and DASS subscales.

\begin{tabular}{|c|c|c|c|}
\hline \multirow{2}{*}{ WHOQOL-BREF } & \multicolumn{3}{|c|}{ DASS } \\
\hline & Depression & Anxiety & Stress \\
\hline Physical Health & $-0.538^{* * *}$ & $-0.507^{* * *}$ & $-0.451^{* * *}$ \\
\hline Psychological Health & $-0.576^{* * *}$ & $-0.460^{* * *}$ & $-0.469^{* * *}$ \\
\hline Social Relationships & $-0.317^{* *}$ & $-0.247^{*}$ & $-0.226^{*}$ \\
\hline Environment & $-0.366^{* * *}$ & $-0.283^{* *}$ & $-0.340^{* *}$ \\
\hline Overall QoL / General Health & $-0.524^{* * *}$ & $-0.444^{* * *}$ & $-0.422^{* * *}$ \\
\hline
\end{tabular}

Table IV. Multiple regression results with WHOQOL-BREF dimensions as dependent variables and DASS subscales as independent, adjusted for patients' characteristics.

\begin{tabular}{|c|c|c|c|c|c|c|}
\hline \multirow{3}{*}{ WHOQOL-BREF } & \multicolumn{6}{|c|}{ DASS } \\
\hline & \multicolumn{2}{|c|}{ Depression } & \multicolumn{2}{|c|}{ Anxiety } & \multicolumn{2}{|c|}{ Stress } \\
\hline & $\beta(\mathbf{S E})^{*}$ & P value & $\beta(\mathrm{SE})^{*}$ & P value & $\beta(\mathbf{S E})^{*}$ & P value \\
\hline Physical Health & $-0.119(0.022)$ & $<0.001$ & $-0.105(0.025)$ & $<0.001$ & $-0.100(0.026)$ & $<0.001$ \\
\hline Psychological Health & $-0.149(0.027)$ & $<0.001$ & $-0.108(0.033)$ & 0.002 & $-0.120(0.033)$ & $<0.001$ \\
\hline Social Relationships & $-0.076(0.029)$ & 0.010 & $-0.047(0.032)$ & 0.144 & $-0.058(0.032)$ & 0.075 \\
\hline Environment & $-0.028(0.024)$ & 0.248 & $-0.013(0.027)$ & 0.631 & $-0.027(0.027)$ & 0.313 \\
\hline Overall QoL / General Health & $-0.099(0.028)$ & 0.001 & $-0.083(0.032)$ & 0.011 & $-0.083(0.032)$ & 0.012 \\
\hline
\end{tabular}

${ }^{*}$ Regression coefficient (standard error) adjusted for socio-demographic and medical characteristics.

Internal consistency reliability of the WHOQOL, using Cronbach's alpha coefficient, was found as 0.90 in "Physical Health", 0.91 in "Psychological Health", 0.81 in Social Relationships, 0.79 in "Environment" and 0.73 in "Overall QoL / General Health". A value of Cronbach's Alpha $(\alpha) \geq 0.70$ reflects good internal consistency reliability of a scale's items. In this case, all WHOQOL domains showed very good reliability (Table III). The scores for "Overall QoL / General Health" ranged from 4.00 to 18.00, with a mean of $12.51(\mathrm{SD}=2.99)$. The median coincides with the value of the midpoint of the response scales, namely the value 12 , indicating that patients were equally divided between high and low QoL self-assessment values and levels of satisfaction with their health status (Table II).

Based on the mean value of the WHOQOL-BREF dimensions (Table II), it seems that social relationship dimensions of QOL showed the highest value (12.40), followed by physical health (11.79), environment (11.71) and psychological health (11.65) dimensions.

Table III presents the results for the relationship between DASS subscales and WHOQOL-BREF dimensions in patients with hematologic malignancies. The investigation of the relationship between DASS scale and QOL showed negative correlations between the DASS anxiety, depression, stress and total scales and all dimensions of patient's quality of life.
A multiple linear regression analysis was performed to explore the relationship between mental health and quality of life among patients with haematological malignancies. Table IV presents the regression coefficients $(\beta)$ for DASS subscales scores in WHOQOL-BREF domains scores after controlling for sociodemographic and medical characteristics, which indicated significant differences in the univariate analyses. The results showed negative impact of mental health disorders on patients' quality of life, except the "environment" dimension. Specifically, depression was independently associated with physical health $(\beta=-0.119$, $\mathrm{p}<0.001)$, psychological health $(\beta=-0.149, \mathrm{p}<0.001)$, social relationships $(\beta=-0.076, p=0.010)$, and overall quality of life / general health $(\beta=-0.099, p=0.001)$. On the other hand, anxiety was negatively related to physical health $(\beta=-$ $0.105, p<0.001)$, psychological health $(\beta=-0.108, p=0.002)$, and overall quality of life / general health $(\beta=-0.083$, $\mathrm{p}=0.011$ ). Finally, stress was independent predicting factor for quality of life related with physical health $(\beta=-0.100$, $\mathrm{p}<0.001)$, psychological health $(\beta=-0.120, \mathrm{p}<0.001)$, and overall quality of life / general health $(\beta=-0.083, p=0.012)$.

\section{Discussion}

Findings from international literature review has revealed that several studies are agreeing with our findings and others that seem to be in disagreement with our results. 
In a study by Koizumi et al. (2018), it was found that patients with hematologic malignancies have particularly high rates of anxiety and depression and often develop negative affect. Moreover, the psychological problems faced by cancer patients impair their quality of life. When cancer patients feel anxious, they tend to direct their attention toward stimuli associated with threat in the environment. If attentional bias occurs in patients with hematopoietic malignancies, who are at particular risk of developing negative effect, resolution of the bias could be useful in relieving their anxiety. Specifically, the study results have shown that attentional bias toward threatening expressions could be positively correlated with the mental level of anxiety and fatigue in patients with hematologic malignancies [14].

In 2018 Poh Loh et al. carried out a survey to investigate the prevalence of sleep disturbance with coexisting depression, fatigue, and pain in elderly cancer patients. They also examined correlations between various socio-demographic and clinical variables and sleep disorders. This cross-sectional study included 389 older patients with solid and hematologic malignancies who were referred to the Specialized Oncology Care \& Research in the Elderly (SOCARE) clinics at the Universities of Rochester and Chicago between May 2011 and October 2015 and completed a sleep and geriatric assessment which included an evaluation of fatigue, pain, and depression. Multivariate logistic regression was used to identify variables associated with sleep problems. The prevalence of sleep disturbance was $40 \%$. $84 \%$ of participants with sleep disturbance $(\mathrm{n}=154)$, also had at least one of the other three symptoms ( $25 \%$ had one symptom, $38 \%$ had two symptoms, and $21 \%$ had three symptoms). Sleep disturbance was more likely to be reported in individuals with comorbidities (45\% vs. $28 \%, \mathrm{P}=0.002$ ), depression ( $49 \%$ vs. $36 \%, \mathrm{P}=0.015$ ), fatigue $(49 \%$ vs. $23 \%, \mathrm{P}<0.001)$, and pain $(45 \%$ vs. $31 \%, \mathrm{P}=0.010$ ). On multivariable analysis, only fatigue (adjusted odds ratio (AOR) 1.90, 95\% CI 1.10-3.30, $\mathrm{P}=0.020$ ) was independently associated with the presence of sleep disturbance. Sleep disturbance is prevalent, and depression, fatigue, or pain are often coexisting symptoms in sleep disturbance among older patients with hematologic malignancies. Fatigue was significantly associated with sleep disturbance, and future research should explore interventions targeting sleep disturbance and fatigue [15].

In a previous study conducted by Haes et al. (1990), the Rotterdam Symptom Checklist (RSCL) was used to assess physical and psychological distress among cancer patients. The structure and stability of the RSCL were examined in three studies. For the validation of the questionnaire, it was first administered to a sample of 95 women with haematological malignancies visiting the clinic either for either chemotherapy or follow-up $(n=86$, $10 \%$ refused to participate). The second of these studies was conducted in 60 patients who participated in a randomized trial comparing two chemotherapy regimens, Hexacaf and CHAP-5, for the treatment of advanced ovarian cancer. The third study compared the quality of life of cancer patients with that of healthy individuals. $78 \%$ of patients and $72 \%$ of normal individuals completed and returned the questionnaire. The psychological dimension proved to be a stable element in all the populations, while the pattern based on the experience of physical distress was less stable. In the first and second studies, three factors have been identified to affect the different areas of symptoms, namely pain, fatigue and gastrointestinal complaints. This distinction was not found in the third study. This difference could be explained by the homogeneity of the populations studied. In the first two studies, most participating patients were receiving chemotherapy. In the heterogeneous population of the third study treatment toxicity and disease experience, which might have been more diverse, were investigated and therefore the relationship between symptoms on which the analysis is based is weaker [16].

\section{Conclusions}

The findings of this study showed that health related Quality of Life in patients hematological undergoing chemotherapy is affected by psychological distress. Qol domains such as physical Health, psychological Health, social Relationships and general Health are negative affected by depression, anxiety and stress in patients with hematological malignancies. Overall, the findings of the study may appear useful in clinical practice. In practical terms, the findings are useful to health professionals, physicians and nurses that are in charge of providing treatment and health care in patients with such malignancies. High levels of depression, anxiety and stress may depict the unfulfilled supportive care needs that hematological cancer patients have. Psychosocial distress can be minimized by specific psychoeducational interventions and programs, which focus on awareness and training on specific mental health components such as depressive and anxiety symptoms, as well as other mental health issues as suicidal ideation.

\section{References}

1. Yfantopoulos J. The "social" quality of life. Arch Hell Med. 2001;18:108-113.

2. Yfantopoulos J. Quality of life and QALYs in the measurement of health. Arch Hell Med. 2001;18:114-130.

3. Vietri J, Otsubo T, Montgomery W, Tsuji T, Harada E. The incremental burden of pain in patients with depression: results of a Japanese survey. BMC Psychiatry. 2015;15:104.

4. Ginieri-Coccossis M, Theofilou P, Synodinou C, Tomaras V, Soldatos C. Quality of life, mental health and health beliefs in haemodialysis and peritoneal dialysis patients: investigating differences in early and later years of current treatment. BMC Nephrol. 2008;9:14. 
5. Theofilou P. Assessing health-related quality of life using the Missoula - Vitas Quality of Life Index (MVQOLI). J Clin Trials. 2012;02:1-2.

6. Lesko LM, Holland JC. Psychological issues in patients with hematological malignancies. Recent Results Cancer Res. 1988;108:243-270.

7. Allart-Vorelli P, Porro B, Baguet F, Michel A, Cousson-Gélie F. Haematological cancer and quality of life: a systematic literature review. Blood Cancer J. 2015;5:e305.

8. Bellizzi KM, Miller MF, Arora NK, Rowland JH. Positive and negative life changes experienced by survivors of nonHodgkin's lymphoma. Ann Behav Med. 2007;34:188-199.

9. McGrath P. Findings on Family Issues During Relocation for Hematology Care. Oncol Nurs Forum. 2015;42:E250-E256.

10. Lovibond PF, Lovibond SH. The structure of negative emotional states: comparison of the Depression Anxiety Stress Scales (DASS) with the Beck Depression and Anxiety Inventories. Behav Res Ther. 1995;33:335-343.

11. Lyrakos GN, Arvaniti C, Smyrnioti M, Kostopanagiotou G. Translation and validation study of the depression anxiety stress scale in the Greek general population and in a psychiatric patient's sample. Abstr 19th Eur Congr Psychiatry 2011;26:1731.

12. The World Health Organization Quality of Life assessment (WHOQOL): position paper from the World Health Organization. Soc Sci Med. 1995;41:1403-1409.

13. Ginieri-Coccossis M, Triantafillou E, Tomaras V, Soldatos C, Mavreas V, Christodoulou G. Psychometric properties of WHOQOL-BREF in clinical and healthy Greek populations: incorporating new culture- relevant items. Psychiatriki. 2012;23:130-142.

14. Koizumi K, Tayama J, Ishioka T, Nakamura-Thomas H, Suzuki M, Hara M, et al. Anxiety, fatigue, and attentional bias toward threat in patients with hematopoietic tumors. PLoS One. 2018;13:e0192056.

15. Loh KP, Zittel J, Kadambi S, Pandya C, Xu H, Flannery M, et al. Elucidating the associations between sleep disturbance and depression, fatigue, and pain in older adults with cancer. J Geriatr Oncol. 2018;9:464-468.

16. de Haes JC, van Knippenberg FC, Neijt JP. Measuring psychological and physical distress in cancer patients: structure and application of the Rotterdam Symptom Checklist. Br J Cancer. 1990;62:1034-1038. 